\title{
Entanglement, fidelity, and quantum phase transition in antiferromagnetic-ferromagnetic alternating Heisenberg chain
}

\author{
Jie Ren and Shiqun Zhu* \\ School of Physical Science and Technology, Suzhou University, \\ Suzhou, Jiangsu 215006, People's Republic of China
}

(Dated: September 2, 2018)

\begin{abstract}
The fidelity and entanglement entropy in an antiferromagnetic-ferromagnetic alternating Heisenberg chain are investigated by using the method of density-matrix renormalization-group. The effect of anisotropy on fidelity and entanglement entropy are investigated. The relations between fidelity, entanglement entropy and quantum phase transition are analyzed. It is found that the quantum phase transition point can be well characterized by both the ground-state entropy and fidelity for large system.
\end{abstract}

PACS numbers: 03.67.-a, 03.65.Ud, 75.10.Pq

Key words: Entropy, Fidelity, DMRG

\footnotetext{
* Corresponding author,E-mail: Szhu@suda.edu.cn
} 
In condense matter physics, quantum phase transitions imply fluctuations, which happened at the zero temperature [1]. When a controlling parameter changes across critical point, some properties of the many-body system will change dramatically. Many results show that entanglement existed naturally in the spin chain when the temperature is at zero. The quantum entanglement of a many-body system has been paid much attention since the entanglement is considered as the heart in quantum information and computation [2, 3]. As the bipartite entanglement measurement in a pure state, the von Neumann entropy[4] in the ferromagnetic [5] and antiferromagnetic [6, 7] spin chains are investigated respectively. By using the cross fields of quantum many-body theory and quantum-information theory, von Neumann entropy is applied to detect quantum critical behaviors [8 12]. A typical example is that Osborne solved exactly one-dimensional infinite-lattice transverse-field Ising model to obtain entropy by the Jordan-Wigner transform. The entropy predicts the quantum phase transition point successfully[9]. Moreover, another concept from quantum information science, ground state fidelity has been used to qualify quantum phase transitions successfully in the last few years [13 22]. It is shown that the fidelity and the entanglement entropy have similar predictive power for identifying quantum phase transitions in the most system. However, the ground state fidelity is a model-dependent indicator for quantum phase transitions. It can not be used to characterize the quantum phase transition in Heisenebeg model with next-nearest-neighbor interavtion for finite-size[23]. Similarly, the fidelity can not detect a BKT-like phase transition, which happens at $\Delta=1$ in antiferromagnetic anisotropic Heisenberg model[23].

Recently, It is reported that $\left(\mathrm{CH}_{3}\right)_{2} \mathrm{CHNH}_{3} \mathrm{CuCl}_{3}$ is realization of the spin-1/2 alternating antiferromagnetic-ferromagnetic $(\mathrm{AF}-\mathrm{F})$ chain by nearly the same strength of the antiferromagnetic and ferromagnetic couplings. There are examples for the alternating AFF spin-1/2 chain compounds such as $[\mathrm{Cu}(\mathrm{TIM})] \mathrm{CuCl}_{4}[24], \mathrm{CuNb}_{2} \mathrm{O}_{6}[25]$ and $\mathrm{DMACuCl}_{3}$ [26]. The ground state properties of the alternating AF-F spin-1/2 chains have been intensely studied[27-29]. Entanglement, fidelity and their relations with quantum phase transition in the system like these materials need to be investigated further.

In this paper, the fidelity and entanglement entropy in the spin- $1 / 2$ alternating AF-F chain with anisotropic interaction are investigated. Firstly, the effect of anisotropic interaction on ground state fidelity is investigated. Secondly, the effect of anisotropic interaction on entropy is calculated. Thirdly, their relations with quantum phase transition are analyzed. 
At last, a discussion concludes the paper.

The Hamiltonian of an antiferromagnetic-ferromagnetic alternating Heisenberg chain with anisotropy of $\mathrm{N}$ sites is given by

$H=J_{A F} \sum_{i=1}^{N / 2}\left(S_{2 i-1}^{x} S_{2 i}^{x}+S_{2 i-1}^{y} S_{2 i}^{y}+\Delta_{A F} S_{2 i-1}^{z} S_{2 i}^{z}\right)+J_{F} \sum_{i=1}^{N / 2}\left(S_{2 i}^{x} S_{2 i+1}^{x}+S_{2 i}^{y} S_{2 i+1}^{y}+\Delta_{F} S_{2 i}^{z} S_{2 i+1}^{z}\right)$,

where $S_{i}^{\alpha}(\alpha=x, y, z)$ are spin operators on the $j$-th site, $N$ is the length of the spin chain. $J_{F}$ and $J_{A F}$ denote the ferromagnetic and antiferromagnetic couplings respectively. $\Delta_{A F}, \Delta_{F}$ are anisotropic interaction. The AF-F alternating spin chain can be regarded as the spin-1 antiferromagnetic chain in the large ferromagnetic coupling limit[27 29]. In the paper, open boundary condition is considered, and we set $J_{A F}=-J_{F}=1, \Delta_{A F}=1$ and $\Delta_{F}>0$.

Ground state fidelity can be applied to detect the existence of the quantum phase transitions. The definition of ground state fidelity is shown as following. A general Hamiltonian of quantum many-body systems can be written as $H(\lambda)=H_{0}+\lambda H_{I}$ where $H_{I}$ is the driving Hamiltonian and $\lambda$ denotes its strength. Supposes $|G s\rangle$ represents the ground state of the system. The ground-state fidelity between $|G s(\lambda)\rangle$ and $|G s(\lambda+\delta)\rangle$ is defined as

$$
F(\lambda, \delta)=|\langle G s(\lambda) \mid G s(\lambda+\delta)\rangle|
$$

Because $F(\lambda, \delta)$ reaches its maximum value $F_{\max }=1$ for $\delta=0$, on expanding the fidelity in powers of $\delta$, the first derivative $\frac{\partial F(\lambda, \delta=0)}{\partial \lambda}=0$. By using the property, the fidelity can written by

$$
F(\lambda, \delta) \simeq 1+\left.\frac{\partial^{2} F(\lambda, \delta)}{2 \partial \lambda^{2}}\right|_{\lambda=\lambda^{\prime}} \delta^{2}
$$

therefore, the average fidelity susceptibility $S(\lambda, \delta)$, is given by [17, 18]

$$
S(\lambda, \delta)=\lim _{\delta \rightarrow 0} \frac{2[1-F(\lambda, \delta)]}{N \delta^{2}} .
$$

It is well known that it is hard to calculate the ground state fidelity because of the lack of knowledge of the ground state function. For models that are not exactly solvable, most of researchers resort to exact diagonalization to obtain the ground state for small size. This method can not precisely quantify the quantum phase transition because the size of 
the system is too small. The method of density-matrix renormalization-group (DMRG) [30, 31] can be applied to obtain the ground state of the model. Moreover, the technology of calculating the overlap of two different ground states by the DMRG has been used for about a decade 32 34]. The method is used to calculate the ground state fidelity susceptibility. We calculate $N$ up to $78 . \delta=0.001$ is used like in Ref.[22]. The total number of density matrix eigenstates held in system block $m=128$ in the basis truncation procedure. The Matlab codes of DMRG with double precision are performed in private computer, and the truncation error is smaller than $10^{-12}$. The fidelity susceptibility $S$ is plotted as a function of anisotropic interaction $\Delta_{F}$ for different sizes in Fig. 1. It is shown that one peak is exist. The maximal value increases with size increases. The location of the maximal value deceases with size increases. When $N=78$, the location of the maximal value $\Delta_{F}^{\max }=2.32$.

For comparison, ground state entanglement entropy is used to detect the quantum phase transition point too. The definition of entanglement entropy is given as follow. Let $|G s\rangle$ be assigned to the ground state of a chain of $N$ qubits, the reduced density matrix of right-hand $L$ contiguous qubits can be written as $\rho_{L}=\operatorname{Tr}_{(N-L)}|G s\rangle\langle G s|$. The bipartite entanglement between the right-hand $L$ contiguous qubits and the rest subsystem can be measured by the entanglement entropy as

$$
E(L, N)=-\operatorname{Tr}\left(\rho_{L} \log _{2} \rho_{L}\right)
$$

By using the method of density-matrix renormalization-group (DMRG), the entropy of ground state for large system can be calculated. The entropy of ground state is plotted as a function of anisotropic interaction $\Delta_{F}$ with sizes of $N=20,40,60,80,100$ with in Fig. 2. The largest states of $m=64$ is kept and $L=N / 2-1$ is considered. There is one peak too. The peak $E_{\max }$ increases with the size increases. The corresponding critical point $\Delta_{F}^{\max }$ of the peak decreases with the size increases.

As we known, the peak in fidelity susceptibility and the peak in entanglement entropy indicate the quantum phase transition. To be more precise, we also investigate whether the positions of the extreme points of entanglement entropy and fidelity in an infinite system. The results for the scaling of entanglement[35] and fidelity [36] can be used to investigate the quantum phase transition point. We plot the maximum entropy of entanglement and fidelity susceptibility as a function of the inverse size of the system. A numeric fit is made, the results is shown in Fig. 3. They apparently agree with linear scaling. In the thermodynamic 
limits, their values tend to $\Delta_{F}^{c}=2.3$, respectively. Both the extreme points of fidelity susceptibility labeled by $S$ and entropy labeled by $E$ represent indeed the quantum phase transition. As we known, when $\Delta_{F}=1$, the phase is the Haldane phase[27-29]. In the limits $\Delta_{F} \rightarrow \infty$, the phase is Ising model universality class state. Our result is similar with the result $\Delta_{A F} / 2 \simeq J_{F} / J_{A F}[29]$. It confirms further that the Haldane-Ising transition occurs at the point.

In the paper, the fidelity susceptibility and entropy in the Heisenberg chain with the Alternating AF-F interaction are studied. By the present DMRG calculations for the model, the effect of anisotropic interaction on fidelity susceptibility and entropy in large size is presented. Their relations with quantum phase transition are investigated. It is shown that the point of the quantum phase transition is clearly marked by the peak of the fidelity susceptibility and entropy. The critical point in the thermodynamic limit is obtained by using the finite-size scaling theory. The fidelity susceptibility and the entanglement entropy can have similar predictive power for revealing quantum phase transition in the system.

\section{Acknowledgments}

It is a pleasure to thank Yinsheng Ling and Jianxing Fang for their many helpful discussions. The financial supports from the Specialized Research Fund for the Doctoral Program

of Higher Education of China (Grant No. 20050285002) and the National Natural Science Foundation of China (Grant No. 10774108) are gratefully acknowledged.

[1] S. Sachdev, Quantum Phase Transitions (Cambridge University Press, Cambridge, England, 1999).

[2] M. A. Nielson and I. L. Chuang, Quantum Computation and Quantum Information (Cambridge University Press, Cambridge, England, 2000).

[3] C. H. Bennett, G. Brassard, C. Crepeau, R. Jozsa, A. Peres, and W. K. Wootters, Phys. Rev. Lett. 70 (1993) 1895.

[4] C. H. Bennett, D. P. DiVincenzo, J. A. Smolin, and W. K. Wootters, Phys. Rev. A 54 (1996) 3824 .

[5] V. Popkov and M. Salerno, Phys. Rev. A 71 (2005) 012301.

[6] G. Vidal, J. I. Latorre, E. Rico, and A. Kitaev, Phys. Rev. Lett. 90 (2003) 227902. 
[7] J. I. Latorre, E. Rico, and G. Vidal, Quant. Inf. Comput. 4 (2004) 48.

[8] J. Preskill, J. Mod. Opt. 47 (2000) 127.

[9] T. J. Osborne, and M. A. Nielsen, Phys. Rev. A 66 (2002) 032110 .

[10] L. Amico, R. Fazio, A. Osterloh, and V. Vedral, Rev. Mod. Phys. 80 (2008) 517.

[11] S. J. Gu, S. S. Deng, Y. Q. Li, and H. Q. Lin, Phys. Rev. Lett. 93 (2004) 086402.

[12] A. Kitaev, and J. Preskill, Phys. Rev. Lett. 96 (2006) 110404.

[13] H. Q. Zhou, J. H. Zhao, and B. Li, arXiv:0704.2940; H. Q. Zhou, arXiv:0704.2945.

[14] P. Zanardi, M. Cozzini, and P. Giorda, arXiv:cond-mat/ 0606130.

[15] N. Oelkers and J. Links, Phys. Rev. B 75 (2007) 115119 .

[16] L. C. Venuti and P. Zanardi, Phys. Rev. Lett. 99 (2007) 095701.

[17] M. Cozzini, R. Ionicioiu, and P. Zanardi, Phys. Rev. B 76 (2007) 104420 .

[18] P. Buonsante and A. Vezzani, Phys. Rev. Lett. 98 (2007) 110601.

[19] W. L. You, Y. W. Li, and S. J. Gu, Phys. Rev. E 76 (2007) 022101.

[20] M. F. Yang, Phys. Rev. B 76 (2007) 180403(R).

[21] H. Q. Zhou, R. Orús, and G. Vidal, Phys. Rev. Lett. 100 (2008) 080601.

[22] Y. C. Tzeng and M. F. Yang, Phys. Rev. A 77 (2008) 012311.

[23] S. Chen, L. Wang, S. J. Gu, and Y. P. Wang, Phys. Rev. E 76 (2007) 061108.

[24] M. Hagiwara, Y. Narumi, K. Kindo, T. C. Kobayashi, H. Yamakage, K. Amaya, and G. Schumauch, J. Phys. Soc. Jpn. 66 (1997) 1792.

[25] K. Kodama, H. Harashina, H. Sasaki, M. Kato, M. Sato, K. Kakurai, and M. Nishi, J. Phys. Soc. Jpn. 68 (1999) 237.

[26] M. B. Stone, W. Tian, M. D. Lumsden, G. E. Granroth, D. Mandrus, J.-H. Chung, N. Harrison, S. E. Nagler, Phys.Rev. Lett. 99 (2007) 087204.

[27] K. Hida, Phys. Rev. B 46 (1992) 8268.

[28] M. Kohmoto and H. Tasaki, Phys. Rev. B 46 (1992) 3486 .

[29] M. Yamanaka, Y. Hatsugai, and M. Kohmoto, Phys. Rev. B 48 (1993) 9555.

[30] S. R. White, Phys. Rev. B 48 (1993) 10345.

[31] U. Schollwöck, Rev. Mod. Phys. 77 (2005) 259.

[32] S. Qin, M. Fabrizio, and L. Yu, Phys. Rev. B 54 (1996) R9643 .

[33] S. Qin, M. Fabrizio, L. Yu, M. Oshikawa, and I. Affleck, Phys. Rev. B 56 (1997) 9766.

[34] I. P. McCulloch, J. Stat. Mech.: Theory Exp. (2007) P10014. 
[35] J. I. Latorre, R. Orus, Phys. Rev. A 69 (2004) 062302.

[36] S. J. Gu, H. M. Kwok, W. Q. Ning, H.Q. Lin Phys. Rev. B 77 (2008) 245109. 


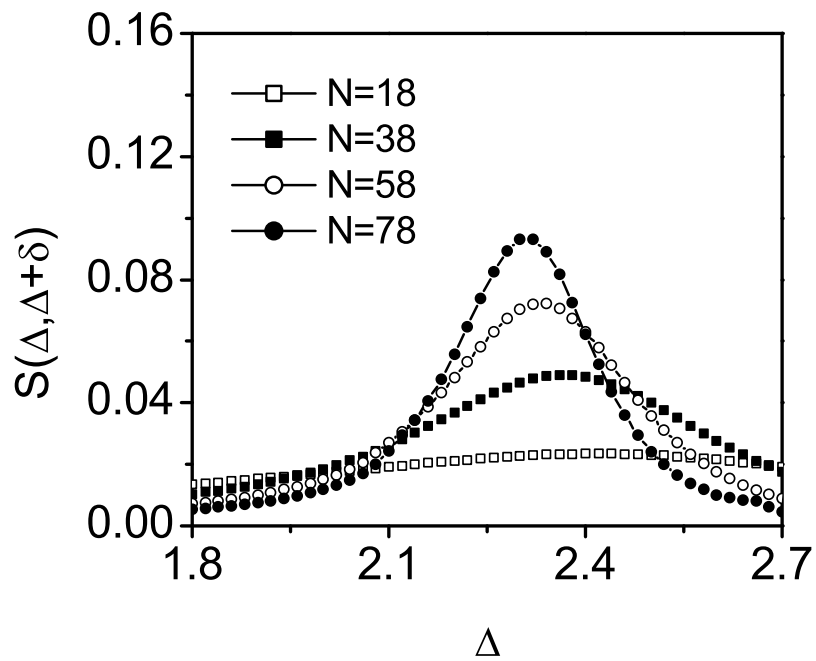

FIG. 1: The fidelity suscepility $S$ is plotted as a function of anisotropic interaction $\Delta_{F}$ for different sizes. 


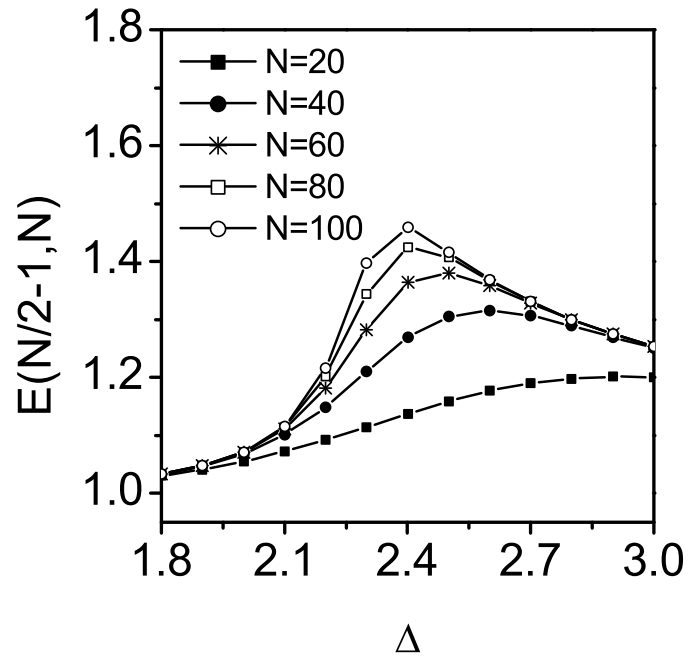

FIG. 2: The entropy $E$ is plotted as a function of anisotropic interaction $\Delta_{F}$ for different sizes. 


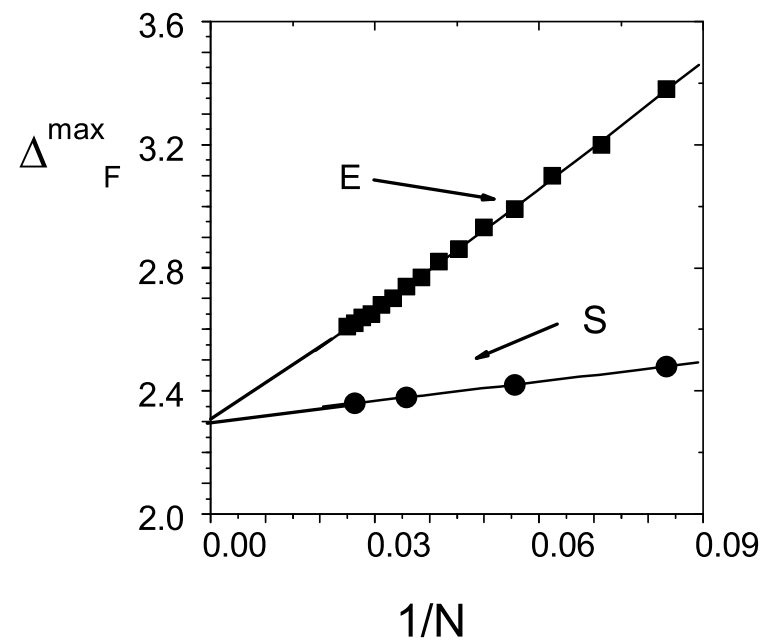

FIG. 3: Scaling of $\Delta_{F}^{\max }$ versus $N^{-1}$. The $\mathbf{\square}$ for entropy and $\bullet$ for fidelity susceptibility are obtained by numeric stimulation and the lines are the fit lines. 